\title{
Color-spin locking phase in two-flavor quark matter for compact star phenomenology
}

\author{
D. N. Aguilera* \\ Institut für Physik, Universität Rostock, \\ Universitätsplatz 3, 18051 Rostock, Germany \\ D. Blaschke $\dagger$ \\ Gesellschaft für Schwerionenforschung (GSI), \\ Planckstr. 1, 64291 Darmstadt, Germany \\ Bogoliubov Laboratory of Theoretical Physics, JINR Dubna, \\ Joliot-Curie Street 6, 141980 Dubna, Russia \\ M. Buballa f \\ Institut für Kernphysik, TU Darmstadt, \\ Schlossgartenstr. 9, 64289 Darmstadt, Germany \\ V.L. Yudichev\$ \\ Bogoliubov Laboratory of Theoretical Physics, JINR Dubna, \\ Joliot-Curie Street 6, 141980 Dubna, Russia
}

\begin{abstract}
We study a spin-1 single flavor color superconducting phase which results from a color-spin locking (CSL) interaction in two-flavor quark matter. This phase is particularly interesting for compact star cooling applications since the CSL phase may survive under charge neutrality constraints implying a mismatch between up- and down-quark chemical potentials which can destroy the scalar diquark condensate. CSL gaps are evaluated within an NJL model and they are found to be consistent with cooling phenomenology if a density dependent coupling constant is used.
\end{abstract}

PACS numbers: $12.38 . \mathrm{Mh}, 24.85 .+\mathrm{p}, 26.60 .+\mathrm{c}, 97.60 . \mathrm{Jd}$

Keywords: Color superconductivity, spin-one condensates, compact star cooling

\section{INTRODUCTION}

Theoretical investigations of the QCD phase diagram at low temperatures $T \ll T_{c}$ and high baryochemical potential $\mu_{B} \sim 1 \mathrm{GeV}$ have recently received new impulses when color superconducting quark matter phases with large diquark pairing gaps of about $100 \mathrm{MeV}$ were suggested within nonperturbative low-energy QCD models [1, 2, 3]. Prior to these works, color superconductivity in quark matter has been discussed using perturbative onegluon exchange interactions resulting in small diquark pairing gaps of about $1 \mathrm{MeV}$ only [4, 5]. More systematic studies of the possible diquark pairing patterns have revealed a very rich phase structure of cold quark matter, see e.g. 6, 7, 8, 9, 10]. The most prominent color superconducting phases with large diquark pairing gaps are the two-flavor scalar diquark condensate (2SC) and the color-flavor locking (CFL) condensate. The latter requires approximate $\mathrm{SU}(3)$ flavor symmetry and occurs therefore only at rather large quark chemical potentials, $\mu_{q} \gtrsim 410-500 \mathrm{MeV}$ [9, 10, 11, 12].

The most favorable places in nature where color superconducting states of matter are expected to occur are the interiors of compact stars, with temperatures well below $1 \mathrm{MeV}$ and central densities exceeding the nuclear saturation density by about one order of magnitude. However,

\footnotetext{
*Electronic address: deborah.aguilera@uni-rostock.de

${ }^{\dagger}$ Electronic address: blaschke@gsi.de

‡Electronic address: michael.buballa@physik.tu-darmstadt.de

$\S$ Electronic address: yudichev@thsun1.jinr.ru
}

a closer examination of the compact star constraints on phases of dense matter reveals that the requirement of charge neutrality [13] may inhibit flavor asymmetric pair condensation in the 2SC phase and even influence the CFL phase border. As the quark chemical potential corresponding to the highest possible central densities may barely reach the above constraint for the occurrence of strange quark matter 14], it is expected that the volume fraction of a strange quark matter phase in a compact star cannot be sufficient to entail observable consequences. The two-flavor quark matter phases, on the other hand, may occupy a large volume [15, 16], but due to the flavor asymmetry the occurrence of the 2SC phase is rather model dependent [9, 10, 17].

Most sensitive observable consequences of quark matter phases in compact stars come from the cooling evolution and it has been demonstrated that the occurrence of unpaired quarks in the core of hybrid stars leads to rapid cooling via the direct Urca process [18, 19, 20]. To describe existing cooling data for isolated compact stars the occurrence of quark pairing in a 2SC phase is not sufficient and a residual weak pairing channel with a small gap $\Delta_{X} \sim 10-100 \mathrm{keV}$ has been introduced phenomenologically 21]. No microscopic calculation for the justification of this $2 \mathrm{SC}+\mathrm{X}$ phase could be presented up to now.

Given this fact and that the 2SC phase is very fragile with respect to the flavor asymmetry, the question arises whether there exist other pairing patterns in two-flavor quark matter which survive compact star constraints and fulfill the requirements from the cooling phenomenology that all quarks shall be paired with the smallest pairing gap being of the order of several keV. Single flavor spin- 
1 pairs are good candidates since they are inert against large splittings in the quark Fermi levels for different flavors occurring due to electroneutrality They have been introduced first in Refs. [22, 23] and their properties have been investigated later more in detail, see [8] and Refs. therein. Recently, a specific spin-1 pairing pattern - the so-called A phase - has been employed to suggest a new mechanism for the origin of pulsar kicks 24]. The idea is based on the fact that the pairing in the A phase is anisotropic, giving rise to a directed neutrino emission during the cooling process. On the other hand, there are still ungapped modes in the A phase, which would again lead to difficulties with cooling phenomenology.

Therefore, in the present work we want to argue in favor of spin-1 pairing in the color spin locked (CSL) phase as a possible candidate. This phase has recently been found to be the most stable spin-1 phase, at least at very high densities [8]. As we will show, as long as the quarks do not become exactly massless, all modes are gapped in the CSL phase. To study this more quantitatively, we present a microscopic calculation within an NJL-type model, thus providing results for future phenomenological applications, like compact star cooling [21].

\section{NJL MODEL FOR THE CSL PHASE}

The CSL pairing pattern we want to study is defined by the equality of three diquark condensates,

$$
\left\langle q_{f}^{T} C \gamma^{3} \lambda_{2} q_{f}\right\rangle=\left\langle q_{f}^{T} C \gamma^{1} \lambda_{7} q_{f}\right\rangle=\left\langle q_{f}^{T} C \gamma^{2} \lambda_{5} q_{f}\right\rangle \equiv \eta_{f},
$$

where $q_{f}$ is a quark field of flavor $f$ with three color and, of course, two spin degrees of freedom. The operators $\lambda_{A}$ are antisymmetric Gell-Mann matrices acting in color space, while $\gamma^{\mu}$ are Dirac matrices, and $C=i \gamma^{2} \gamma^{0}$ is the matrix of charge conjugation. Hence, each of the three diquark condensates belongs to the antisymmetric antitriplet in color space and, at the same time, represents a vector component in spin-space. This means, both, color $S U(3)$ and rotational $O(3)$, are broken for $\eta_{f} \neq 0$. There remains, however, a residual invariance under a common transformation in which color and spin rotations are locked to each other.

Together with the diquark condensates, we consider the presence of the quark-antiquark condensates

$$
\sigma_{f}=\left\langle\bar{q}_{f} q_{f}\right\rangle,
$$

which are responsible for dynamical chiral symmetry breaking in vacuum. In the density regime we are interested in, these condensates are relatively small. However, as we will see, it is important to keep them in the calculations.

In order to investigate the properties of this condensation pattern, we consider a two-flavor system of quarks, $q=(u, d)^{T}$, and employ an NJL-type Lagrangian

$$
\mathcal{L}_{\text {eff }}=\mathcal{L}_{0}+\mathcal{L}_{q \bar{q}}+\mathcal{L}_{q q},
$$

with a free part

$$
\mathcal{L}_{0}=\bar{q}(i \not \partial-m) q,
$$

and 4-point interactions in the channels corresponding to Eqs. (11) and (2):

$$
\begin{aligned}
& \mathcal{L}_{q \bar{q}}=G \sum_{a=0}^{3}\left(\bar{q} \tau_{a} q\right)^{2}+\ldots, \\
& \mathcal{L}_{q q}=-H_{v}\left(\bar{q} \gamma^{\mu} C \tau_{\mathrm{S}} \lambda_{\mathrm{A}} \bar{q}^{T}\right)\left(q^{T} C \gamma_{\mu} \tau_{\mathrm{S}} \lambda_{\mathrm{A}} q\right)+\ldots
\end{aligned}
$$

Here the matrices $\tau_{a}, a=1,2,3$, are the usual Pauli matrices in flavor space while $\tau_{0}$ is a unit matrix. As before, Gell-Mann matrices in color space are symbolized by $\lambda$. The subscripts $\mathrm{A}$ and $\mathrm{S}$ denote antisymmetric and symmetric matrices, respectively, i.e., $\tau_{\mathrm{S}} \in\left\{\tau_{0}, \tau_{1}, \tau_{3}\right\}$ and $\lambda_{\mathrm{A}} \in\left\{\lambda_{2}, \lambda_{5}, \lambda_{7}\right\}$. As usual, repeated indices are summed over.

$\mathcal{L}_{q \bar{q}}$ and $\mathcal{L}_{q q}$ should be interpreted as effective interactions to be used in mean-field (Hartree) approximation. In general, there could be further interaction terms, indicated by the ellipsis in Eqs. (5) and (6). For instance, $\mathcal{L}_{q q}$ could contain a scalar term in the flavor singlet color antitriplet channel, corresponding to standard 2SC pairing. However, as outlined in the Introduction, we assume that under compact star conditions the mismatch between up and down quark Fermi momenta is too large to allow for cross-flavor condensation. In this case, the corresponding interaction terms do not contribute to the mean-field thermodynamics. In the same way, we assume that there is no significant condensation in any other channel except $\eta_{f}$ and $\sigma_{f}$.

As a specific example, we could think of $\mathcal{L}_{q \bar{q}}$ and $\mathcal{L}_{q q}$ to arise via a Fierz transformation from a color-current interaction $\mathcal{L}_{\text {int }}=-g\left(\bar{q} \gamma^{\mu} \lambda_{a} q\right)\left(\bar{q} \gamma_{\mu} \lambda_{a} q\right)$, corresponding to the quantum numbers of a single gluon exchange. In this case the ratio of the two coupling constants is given by $G: H_{v}=1: \frac{3}{8}$. Later, we will employ this relation in order to constrain the number of parameters in the numerical calculations. At the present stage, however, we can leave $G$ and $H_{v}$ as arbitrary constants.

In order to derive the mean-field thermodynamic potential for an ensemble of up and down quarks at temperature $T$ and chemical potentials $\mu_{u}$ and $\mu_{d}$, respectively, we linearize $\mathcal{L}_{\text {eff }}$ in the presence of the condensates $\eta_{f}$ and $\sigma_{f}$. One finds that the different flavors decouple, i.e., the thermodynamic potential is given by the sum

$$
\Omega_{q}\left(T,\left\{\mu_{f}\right\}\right)=\Omega_{u}\left(T, \mu_{u}\right)+\Omega_{d}\left(T, \mu_{d}\right) .
$$

Defining the constituent quark masses and the diquark gaps

$$
M_{f}=m_{f}-4 G \sigma_{f}, \quad \Delta_{f}=4 H_{v} \eta_{f},
$$

and using the Nambu-Gorkov bispinors

$$
\psi=\frac{1}{\sqrt{2}}\left(\begin{array}{c}
q \\
C \bar{q}^{T}
\end{array}\right), \quad \bar{\psi}=\frac{1}{\sqrt{2}}\left(\bar{q}, q^{T} C\right)
$$

the contributions for each flavor can be written as

$$
\begin{aligned}
\Omega_{f}\left(T, \mu_{f}\right) & =-T \sum_{n} \int \frac{d^{3} p}{(2 \pi)^{3}} \frac{1}{2} \operatorname{Tr} \ln \left(\frac{1}{T} S_{f}^{-1}\left(i \omega_{n}, \vec{p}\right)\right) \\
& +\frac{1}{8 G}\left(M_{f}-m\right)^{2}+\frac{3}{8 H_{v}}\left|\Delta_{f}\right|^{2}
\end{aligned}
$$


where the sum is over fermionic Matsubara frequencies $\omega_{n}=(2 n+1) \pi T$. The inverse of fermion propagator is given by

$$
S_{f}^{-1}(p)=\left(\begin{array}{cc}
\not p+\mu_{f} \gamma^{0}-M_{f} & \hat{\Delta}_{f} \\
-\hat{\Delta}_{f}^{\dagger} & \not p-\mu_{f} \gamma^{0}-M_{f}
\end{array}\right)
$$

in Nambu-Gorkov space, where $\hat{\Delta}_{f}=\Delta_{f}\left(\gamma^{3} \lambda_{2}+\gamma^{1} \lambda_{7}+\right.$ $\gamma^{2} \lambda_{5}$ ). Taking into account its color and Dirac structure, this is a $24 \times 24$ matrix. Explicit evaluation of the trace yields

$\operatorname{Tr} \ln \left(\frac{S_{f}^{-1}(p)}{T}\right)=2\left(\ln \frac{F_{f}^{-}(p)}{T^{2}}+\ln \frac{F_{f}^{+}(p)}{T^{2}}+\ln \frac{G_{f}(p)}{T^{8}}\right)$,

where

$$
F_{f}^{\mp}(p)=p_{0}^{2}-\left(\varepsilon_{f}^{2}+\mu_{f}^{2}+\left|\Delta_{f}\right|^{2} \mp 2 \sqrt{\mu_{f}^{2} \varepsilon_{f}^{2}+\left|\Delta_{f}\right|^{2} \vec{p}^{2}}\right)
$$

and

$$
\begin{aligned}
G_{f}(p) & =\left(p_{0}^{2}-\left(\varepsilon_{f}-\mu_{f}\right)^{2}\right)^{2}\left(p_{0}^{2}-\left(\varepsilon_{f}+\mu_{f}\right)^{2}\right)^{2} \\
& -10\left|\Delta_{f}\right|^{2} A\left(p_{0}^{2}-\left(\varepsilon_{f}-\mu_{f}\right)^{2}\right)\left(p_{0}^{2}-\left(\varepsilon_{f}+\mu_{f}\right)^{2}\right) \\
& -33\left|\Delta_{f}\right|^{4} B_{+} B_{-}-40\left|\Delta_{f}\right|^{6} A+16\left|\Delta_{f}\right|^{8} .
\end{aligned}
$$

Here, we have introduced the abbreviations $\varepsilon_{f}^{2}=\vec{p}^{2}+M_{f}^{2}$ and

$$
\begin{aligned}
A & =p_{0}^{2}-\frac{3}{5} \vec{p}^{2}-\mu_{f}^{2}-M_{f}^{2} \\
B_{ \pm} & =p_{0}^{2}-\frac{25}{33} \vec{p}^{2}-\mu_{f}^{2}-M_{f}^{2} \\
& \pm \frac{2}{33} \sqrt{16 \vec{p}^{4}+297 \mu_{f}^{2} \vec{p}^{2}+561 M_{f}^{2} \mu_{f}^{2}} .
\end{aligned}
$$

Finally, we have to turn out the Matsubara sum. To that end we determine the zeros $E_{f ; k}^{2}$ of the polynomials $F_{f}^{\mp}$ and $G_{f}$ with respect to $p_{0}^{2}$,

$$
\begin{aligned}
& F_{f}^{\mp}\left(p_{0}, \vec{p}\right)=p_{0}^{2}-E_{f ; 1,2}^{2}, \\
& G_{f}\left(p_{0}, \vec{p}\right)=\prod_{k=3}^{6}\left(p_{0}^{2}-E_{f ; k}^{2}\right),
\end{aligned}
$$

and apply the formula

$$
T \sum_{n} \ln \left(\frac{1}{T^{2}}\left(\omega_{n}^{2}+\lambda_{k}^{2}\right)\right)=\lambda_{k}+2 T \ln \left(1+e^{-\lambda_{k} / T}\right)
$$

to get the result

$$
\begin{aligned}
\Omega_{f}\left(T, \mu_{f}\right) & =\frac{1}{8 G}\left(M_{f}-m\right)^{2}+\frac{3}{8 H_{v}}\left|\Delta_{f}\right|^{2} \\
& -\sum_{k=1}^{6} \int \frac{d^{3} p}{(2 \pi)^{3}}\left(E_{f ; k}+2 T \ln \left(1+e^{-E_{f ; k} / T}\right)\right) .
\end{aligned}
$$

This expression is the basis for the further analysis which will be performed on the mean-field level, i.e. at the stationary points

$$
\frac{\delta \Omega_{f}}{\delta \Delta_{f}}=0, \quad \frac{\delta \Omega_{f}}{\delta M_{f}}=0,
$$

defining a set of gap equations for $\Delta_{f}$ and $M_{f}$. Among the solutions, the stable one is the solution which corresponds to the absolute minimum of $\Omega_{f}$.

\section{DISPERSION LAWS}

The six functions $E_{f, k}$, defined in Eqs. (17) and (18) correspond to the dispersion laws of the quasiparticle states of flavor $f$. In the above discussions we have assumed that we know these functions. This is obviously the case for $E_{f ; 1}$ and $E_{f ; 2}$ which are directly read off from Eq. (13):

$$
E_{f ; 1,2}=\sqrt{\varepsilon_{f}^{2}+\mu_{f}^{2}+\left|\Delta_{f}\right|^{2} \mp 2 \sqrt{\mu_{f}^{2} \varepsilon_{f}^{2}+\left|\Delta_{f}\right|^{2} \vec{p}^{2}}} .
$$

Here we have chosen the positive square-root, corresponding to the quasiparticle energy, while the negative square-root corresponds to a quasihole state.

It is advantageous to define effective quantities,

$$
\begin{aligned}
\varepsilon_{f, \mathrm{eff}}^{2} & =\vec{p}^{2}+M_{f, \mathrm{eff}}^{2}, \\
M_{f, \mathrm{eff}} & =\frac{\mu_{f}}{\mu_{f, \mathrm{eff}}} M_{f}, \\
\mu_{f, \mathrm{eff}}^{2} & =\mu_{f}^{2}+\left|\Delta_{f}\right|^{2},
\end{aligned}
$$

and

$$
\Delta_{f, \text { eff }}=\frac{M_{f}}{\mu_{f, \text { eff }}}\left|\Delta_{f}\right|
$$

in order to bring Eq. (22) into the standard form

$$
E_{f ; 1,2}=\sqrt{\left(\varepsilon_{f, \mathrm{eff}} \mp \mu_{f, \mathrm{eff}}\right)^{2}+\Delta_{f, \mathrm{eff}}^{2}} .
$$

Note that, in contrast to the gap parameter $\Delta_{f}$, the effective gap $\Delta_{f \text {,eff }}$ corresponds to the true gap in the spectrum of the first quasiparticle mode. In fact, as we will see below, it is the smallest gap of all quasiparticle modes in our model. It is therefore the relevant quantity for cooling and other transport properties.

Compared with $\Delta_{f}$, the effective gap $\Delta_{f \text {,eff }}$ is suppressed by an additional factor of the order $M_{f} / \mu_{f}$. This implies that the mode $E_{f, 1}$ becomes gapless in the chiral limit when $M_{f}=0$. This is the reason why the current quark mass $m$ and the chiral condensate $\sigma_{f}$ should be retained, even if they are small.

The four remaining dispersion relations $E_{f ; k}, k=$ $3 \ldots 6$ are the zeros of the fourth-order polynomial $G_{f}(p)$ (14). Although, in principle, the exact solutions can be determined analytically, the resulting expressions are extremely long and difficult to handle. In practice, it is therefore more convenient to perform a numerical search.

Alternatively, we can construct approximate analytical solutions which have the advantage that they are more transparent. Anticipating that $\Delta_{f}$ will be small, we expand $E_{f ; k}^{2}$ in powers of $\left|\Delta_{f}\right|^{2}$ and insert this into Eq. (18):

$$
G_{f}\left(p_{0}, \vec{p}\right)=\prod_{k=3}^{6}\left(p_{0}^{2}-\sum_{j=0}^{\infty} c_{f ; k}^{(j)}\left|\Delta_{f}\right|^{2 j}\right)
$$


The coefficients $c_{f ; k}^{(j)}$ can be determined comparing the r.h.s. of this expression order by order with Eq. (14). At lowest order one recovers the free dispersion laws for particles and antiparticles, $c_{f ; 3,5}^{(0)}=\left(\varepsilon_{f}-\mu_{f}\right)^{2}$ and $c_{f ; 4,6}^{(0)}=\left(\varepsilon_{f}+\mu_{f}\right)^{2}$, while the coefficients for the first nontrivial correction are obtained as solutions of quadratic equations. Therefore, the particle branch and the antiparticle branch split:

$$
\begin{aligned}
& E_{f ; 3,5}^{2}=\left(\varepsilon_{f}-\mu_{f}\right)^{2}+c_{f ; 3,5}^{(1)}\left|\Delta_{f}\right|^{2}+\ldots, \\
& E_{f ; 4,6}^{2}=\left(\varepsilon_{f}+\mu_{f}\right)^{2}+c_{f ; 4,6}^{(1)}\left|\Delta_{f}\right|^{2}+\ldots,
\end{aligned}
$$

where

$$
\begin{aligned}
& c_{f ; 3,5}^{(1)}=\frac{1}{2}\left[5-\frac{\vec{p}^{2}}{\varepsilon_{f} \mu_{f}} \pm \sqrt{\left(1-\frac{\vec{p}^{2}}{\varepsilon_{f} \mu_{f}}\right)^{2}+8 \frac{M_{f}^{2}}{\varepsilon_{f}^{2}}}\right], \\
& c_{f ; 4,6}^{(1)}=\frac{1}{2}\left[5+\frac{\vec{p}^{2}}{\varepsilon_{f} \mu_{f}} \pm \sqrt{\left(1+\frac{\vec{p}^{2}}{\varepsilon_{f} \mu_{f}}\right)^{2}+8 \frac{M_{f}^{2}}{\varepsilon_{f}^{2}}}\right] .
\end{aligned}
$$

For illustration, we have plotted the coefficients $c_{f ; k}^{(1)}, k=$ 3...6, in Fig. 1 as functions of $|\vec{p}| / \mu_{f}$. We have chosen a typical chemical potential $\mu_{f}=400 \mathrm{MeV}$ and a typical constituent mass $M_{f}=30 \mathrm{MeV}$. For comparison we also show the result for $M_{f}=0$. Except for small momenta, the mass effect is rather small.

As we will see in the next section, the gap parameter $\Delta_{f}$ is of the order $0.1-10 \mathrm{MeV}$ in our model. We have checked numerically that Eq. (29), neglecting terms of order $\left|\Delta_{f}\right|^{4}$, is sufficiently accurate in this case. We can make use of this to perform a closer examination of the excitation gaps in the spectrum:

Since $\Delta_{f}$ is small, the smallest gaps are expected in the vicinity of the Fermi surface. Evaluating Eqs. (29) and (30) at the Fermi momentum, one obtains

$$
E_{f ; 3,5}^{2}\left(\vec{p}^{2}=\mu_{f}^{2}-M_{f}^{2}\right) \simeq\left(2 \pm \sqrt{2} \frac{M_{f}}{\mu_{f}}\right)\left|\Delta_{f}\right|^{2},
$$

where we have neglected higher orders in $M_{f} / \mu_{f}$. (The antiparticle modes $E_{f ; 4,6}$ are of course irrelevant for this discussion.) Hence, the excitation gaps are considerably larger than $\Delta_{f \text {,eff }}$, which is related to $E_{f ; 1}$, see Eq.(26). In particular, none of the dispersion laws $E_{f ; 3-6}$ can become gapless.

One still might worry about the fact that we have evaluated the energy only at the Fermi momentum. Performing an "exact" minimization, the position of the minimum becomes shifted. However, this shift in momentum is of the order $\left|\Delta_{f}\right|^{2}$, which leads to a correction in the excitation energy of the order $\left|\Delta_{f}\right|^{4}$, i.e., outside the range of validity of our approximation. For instance, for $M_{f}=0$, the minimum of $E_{f ; 5}^{2}$ is located at the momentum $|\vec{p}|=\mu_{f}+\left|\Delta_{f}\right|^{2} /\left(2 \mu_{f}\right)$. At this point one finds $E_{f ; 5}^{2}=2\left|\Delta_{f}\right|^{2}-\left|\Delta_{f}\right|^{4} /\left(4 \mu_{f}^{2}\right)$. Of course, the last term should not be trusted. But even if we did, we would find a gapless solution only if $\left|\Delta_{f}\right| \geq \sqrt{8} \mu_{f}$. This is not only outside the range of our approximations, but also far away from any realistic value of $\left|\Delta_{f}\right|$.

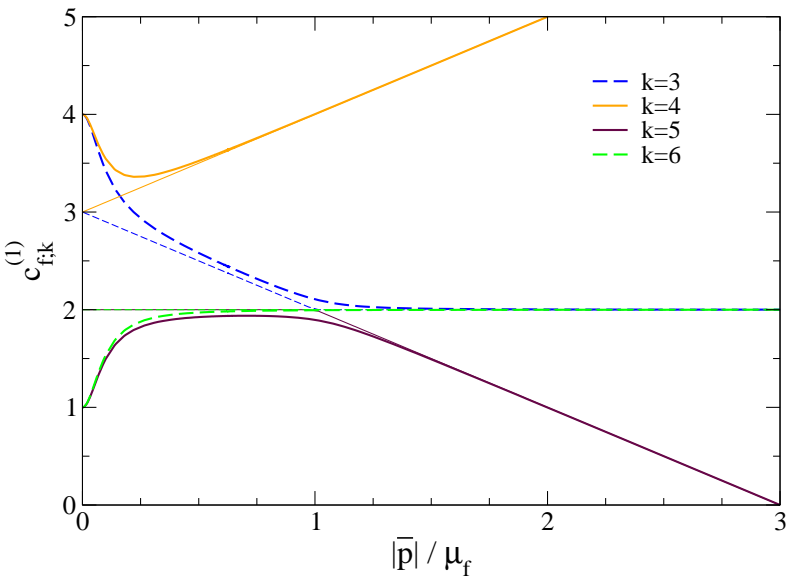

FIG. 1: Dispersion relation coefficients $c_{f ; k}^{(1)}, k=3 \ldots 6$, as functions of $|\vec{p}| / \mu_{f}$ for fixed $\mu_{f}=400 \mathrm{MeV}$. The thick lines correspond to a typical dynamical mass of $M_{f}=30 \mathrm{MeV}$, the thin lines to $M_{f}=0$.

\section{NUMERICAL RESULTS}

\section{A. NJL model}

As we have seen, in our model the different flavors decouple. Therefore, the solutions of the gap equations can be discussed for a single flavor as functions of the corresponding chemical potential $\mu_{f}$. Later, we may combine the solutions for up and down quarks (together with leptons) to obtain electrically neutral matter. In this article, all calculations are performed at $T=0$.

We solve the gap equations using the dispersion relations (27) for the modes 1 and 2 and the approximate dispersion relations (29) for the modes 3-6, where we neglect the terms of order $\left|\Delta_{f}\right|^{4}$. The parameter sets (current quark mass $m$, coupling constant in the meson channel $G$, and three dimensional cut-off $\Lambda$ ) are shown in Tab. I and have been determined by fitting the pion mass and the pion decay constant to their empirical values. They correspond to the vacuum constituent quark masses $M_{f}=300 \mathrm{MeV}, 350 \mathrm{MeV}$, and $400 \mathrm{MeV}$, respectively. For the diquark coupling constant we employ the color-current relation $H_{v}=3 G / 8$.

\begin{tabular}{|c||c|c|c|}
\hline Set & $\Lambda[\mathrm{MeV}]$ & $G \Lambda^{2}$ & $m[\mathrm{MeV}]$ \\
\hline 1 & 664.3 & 2.06 & 5.00 \\
2 & 613.4 & 2.25 & 5.42 \\
3 & 587.9 & 2.44 & 5.60 \\
\hline
\end{tabular}

TABLE I: Parameter sets for NJL model.

In Fig. 2 we show the dynamical quark mass and the 


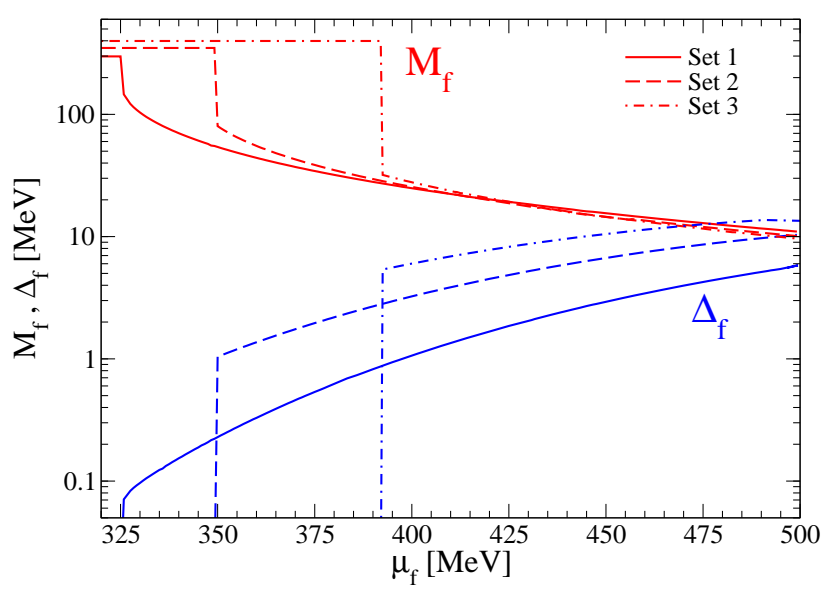

FIG. 2: Dependence of the dynamical quark mass $M_{f}$ and the CSL diquark gap $\Delta_{f}$ on the chemical potential $\mu_{f}$ of the quark flavor $f$ for different parametrizations of the NJL model.

diquark gap as functions of $\mu_{f}$. Obviously, with increasing constituent mass in vacuum, the critical chemical potentials $\mu_{f \text {,crit }}$ for the chiral phase transition also become larger. For all practical purposes, $\mu_{f \text {,crit }}$ also marks the onset of the CSL phase. ${ }^{1}$ Here the size of the gap $\Delta_{f}\left(\mu_{f, \text { crit }}\right)$ increases from Set 1 to Set 3 since the dimensionless coupling constant $H_{v} \Lambda^{2}$ becomes larger.

The CSL gaps are strongly $\mu_{f}$-dependent functions in the considered domain and we easily identify two regimes: while the asymptotic behavior for large density is quite similar for all the sets used $\left(\Delta_{f}\left(\mu_{f, \max }\right) \simeq 10 \mathrm{MeV}\right)$, the low density region is qualitatively determined by the parametrization $\left(\Delta_{f}\left(\mu_{f, \text { crit }}\right) \simeq 0.1-6 \mathrm{MeV}\right.$ depending on the set). In particular for Set 1 we obtain that the superconducting gap has a very low value at $\mu_{f \text {,crit }}$ and then rises by two orders of magnitude up to $\mu_{f}=500 \mathrm{MeV}$ (Fig. [2). For the other sets the corresponding increment is at least one order of magnitude smaller.

The gap functions in the low-density region are crucial in the derivation of equations of state for compact stars applications. Models with high $\mu_{f, \text { crit }}$ like Set 3 might not be able to stabilize hybrid stars configurations [25].

In Fig. [3] we show the results of the effective gaps $\Delta_{f, \text { eff }}$ defined in (26) for the dispersion relations (27) as func-

\footnotetext{
${ }^{1}$ For Set 2 and Set $3, \mu_{f, \text { crit }}$ is smaller than the vacuum constituent mass. Consequently, the density at $T=0$ is zero for $\mu_{f}<\mu_{f, \text { crit }}$, and, thus, there are no quarks which could pair. This is not quite the case for Set 1 . Here $\mu_{f, \text { crit }}$ is larger than the vacuum constituent mass and, hence, there is a small interval below $\mu_{f \text {,crit }}$ where the density is non-zero. In this interval, the attractive quark-quark interaction causes a Cooper instability leading to a non-zero CSL-condensate. However, since the density is very small, the condensate is tiny and practically undetectable with our numerical methods. In any case, the existence of a low-density gas of unconfined constituent quarks is an artifact of the model and has no physical meaning.
}

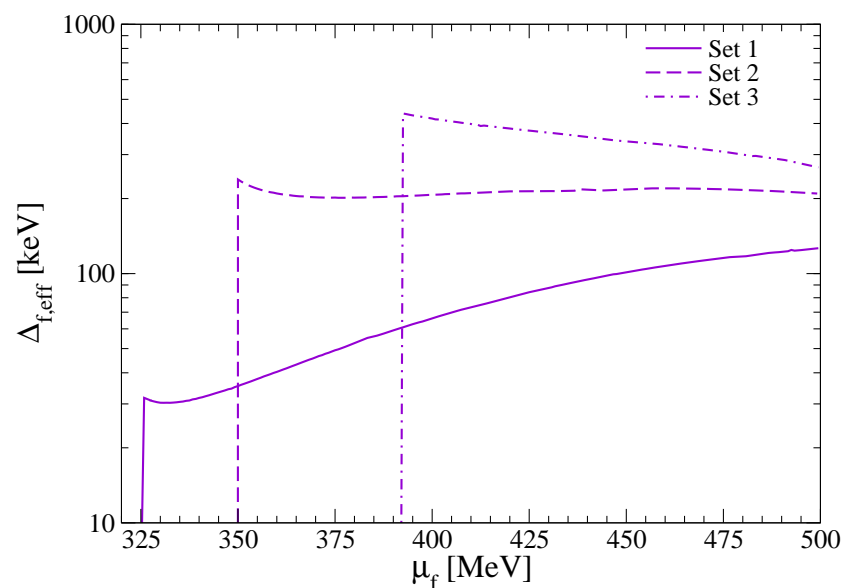

FIG. 3: Effective CSL pairing gap $\Delta_{f \text {,eff }}$, Eq. (26), as function of the chemical potential $\mu_{f}$ for different parametrizations of the NJL model.

tions of $\mu_{f}$. As discussed in Sect. III these are the smallest gaps in the spectrum and thus the relevant quantities for the compact star cooling phenomenology. As expected, the size of $\Delta_{f \text {,eff }}$ is up to two orders of magnitude smaller than $\Delta_{f}$ (cf. Fig. 2). We also observe that the strictly rising density dependence of the latter can partially be compensated by the drop of the dynamical quark mass $M_{f}$ and the increase of $\mu_{f}$. The only exception is found for Set 1 , where $\Delta_{f}$, as described before, has an early onset and rises too strongly. Therefore, we obtain that $\Delta_{f \text {,eff }}\left(\mu_{f}\right)$ is a function which increases by one order of magnitude for Set 1, is nearly constant for Set 2 and even decreasing for Set 3.

We stress once again that the asymptotic behavior of the gaps at $\mu_{f, \max }$ is quite similar while the behavior at $\mu_{f, \text { crit }}$ varies strongly for the different parameter sets.

\section{B. Density dependent coupling}

To obtain a reasonable description of modern cooling data, the pairing pattern for quark matter in hybrid star cores should fulfill a list of constraints, as it has been derived in a recent analysis [21]. These were:

1. All quarks need to be paired (which excludes, e.g., the pure 2SC phase).

2. The smallest gaps should be in the range $10-$ $100 \mathrm{keV}$ (which excludes, e.g., the CFL phase).

3. The smallest gaps should have a decreasing density dependence in the relevant domain of chemical potentials $\mu_{\text {crit }}<\mu \lesssim 500 \mathrm{MeV}$.

We have shown in the previous subsection that the first two conditions are fulfilled for the CSL phase, but the third one is not. Therefore, to qualify the CSL phase 
discussed in this paper as a good candidate for the phase structure of quark matter in compact stars from the point of view of cooling phenomenology, we would like to study here a small modification of the model which could render at least the smallest of the CSL gaps a decreasing function of the density.

Motivated by the logarithmic momentum dependence of the 1-loop running coupling constant $\alpha\left(\vec{p}^{2}\right) \simeq$ $1 / \ln \left(\vec{p}^{2} / \Lambda_{\mathrm{QCD}}^{2}\right)$ and by the fact that near the Fermi surface we have $|\vec{p}| \simeq p_{F}^{f} \simeq \mu_{f}$, we suggest to introduce a density dependence of the couplings according to

$$
G\left(\mu_{f}\right)=G R_{\Lambda_{\mathrm{QCD}}}\left(\mu_{f}\right), \quad H_{v}\left(\mu_{f}\right)=H_{v} R_{\Lambda_{\mathrm{QCD}}}\left(\mu_{f}\right) .
$$

Here, the ratio

$$
R_{\Lambda_{\mathrm{QCD}}}\left(\mu_{f}\right)=\frac{\alpha\left(\mu_{f}\right)}{\alpha\left(\mu_{f, \mathrm{crit}}\right)}=\frac{\ln \left(\mu_{f, \mathrm{crit}} / \Lambda_{\mathrm{QCD}}\right)}{\ln \left(\mu_{f} / \Lambda_{\mathrm{QCD}}\right)},
$$

relates the running coupling constant for $\mu_{f}$ to the value at $\mu_{f, \text { crit }}$. Of course, this formula, which has to be taken as very schematic, cannot be used at arbitrary small chemical potentials. In our calculations, we assume that it is valid for $\mu_{f} \geq \mu_{f, \text { crit }}$ and take $G$ and $H_{v}$ at $\mu_{f}=\mu_{f, \text { crit }}$ from Table $\llbracket \Lambda_{\mathrm{QCD}}$ is the QCD momentum scale which is not well known for the case $N_{f}=2$. We will consider it as a free parameter to be varied in the limits $\Lambda_{\mathrm{QCD}}=210-300 \mathrm{MeV}$.

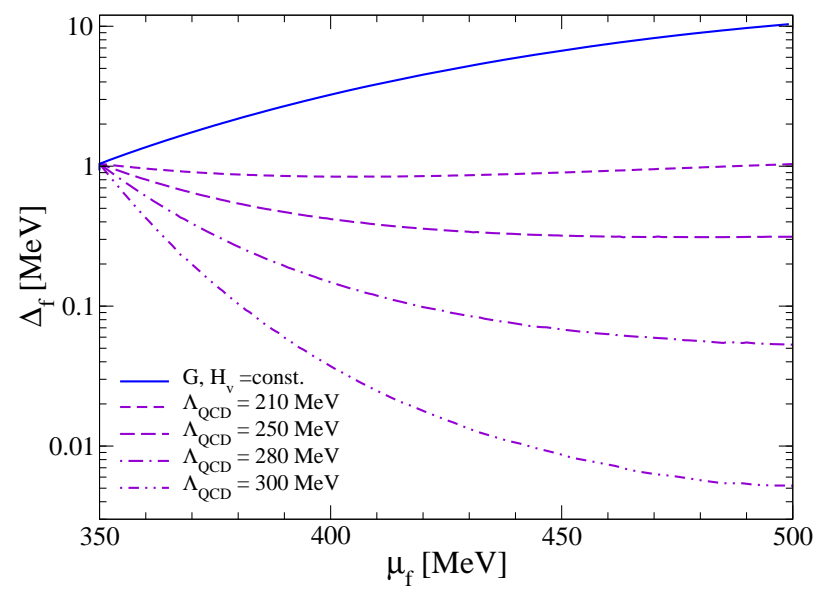

FIG. 4: CSL pairing gap $\Delta_{f}\left(\mu_{f}\right)$ for different density dependent coupling functions $G\left(\mu_{f}\right), H_{v}\left(\mu_{f}\right)$ as defined in Eq. 32 for Set $2\left(\mu_{f, \text { crit }}=350 \mathrm{MeV}\right)$. The solid line represents the case for constant $H_{v}=3 G / 8$. The dashed and dash-dotted lines are for density dependent coupling for different values of $\Lambda_{\mathrm{QCD}}$.

In Fig. 团 we show the CSL gaps for different $\mu_{f^{-}}$ dependent coupling functions for the Set 2 with $\mu_{\text {crit }}=$ $350 \mathrm{MeV}$. We see that the pairing gaps for $\Lambda_{\mathrm{QCD}}=$ $280-300 \mathrm{MeV}$ are decreasing with $\mu_{f}$. The corresponding effective gaps shown in Fig. [5 change by more than two orders of magnitude in the relevant range of chemical potentials, as it is required from cooling phenomenology calculations [21].

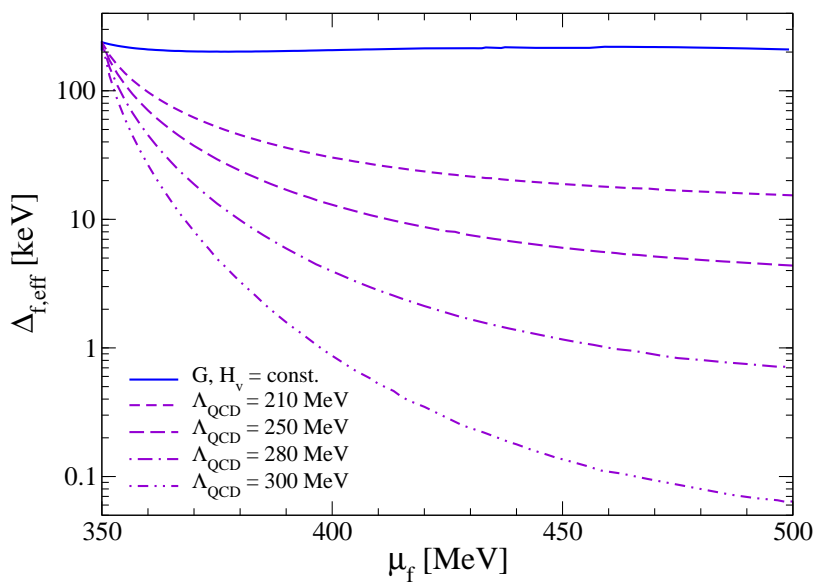

FIG. 5: Same as Fig. 4 for the effective CSL gaps

\section{CONCLUSION}

We have employed an NJL-type model to study a spin1 single-flavor color superconducting phase which results from a color-spin locking (CSL) pairing pattern in twoflavor quark matter. This CSL phase is particularly interesting for compact star cooling applications since it may survive under charge neutrality constraints implying a sufficiently large mismatch between up- and down-quark chemical potentials to destroy the otherwise dominant scalar diquark condensate of the $2 \mathrm{SC}$ phase.

In the specific model we used, the different flavors decouple and could be studied separately. It turned out to be important to retain non-zero quark masses, even if they are small, because the size of the smallest gap in the spectrum is controlled by the value of the density dependent dynamical quark mass.

Hybrid star cooling phenomenology has suggested earlier that quark matter in a compact star should be in a color-superconducting state with all quarks gapped. The smallest gap in the spectrum has to be in the range of several $\mathrm{keV}$ and should decrease with density. Introducing a density dependent coupling, motivated by the running of the QCD coupling constant, we could show that CSL effective gaps can be brought in agreement with these requirements. It would be interesting to see, whether this result can also be obtained within a more microscopic approach, e.g., using Dyson-Schwinger formalism.

Of course, before applying these results to compact stars, we must construct electrically neutral quark matter in beta equilibrium. However, since there is no pairing between different flavors, this does not lead to major complications. In particular, the magnitude of the CSL gaps should not change considerably. Therefore, since the latter nicely matches the requirements from the cooling phenomenology, one is encouraged to consider the CSL quark matter phase as a viable candidate for the superdense matter in the interior of compact stars. 
Another interesting question concerns the possible electromagnetic Meissner effect 5, 26] in the CSL phase [27] in response to a strong magnetic field typical for neutron stars. A more detailed discussion of this issue is, however, beyond the scope of the present work.

\section{Acknowledgment}

We are grateful to H. Grigorian, D. K. Hong, D. Rischke, T. Schäfer, A. Schmitt, I. Shovkovy, D. Voskresensky and Q. Wang for their comments and interest in our work. We acknowledge discussions during the meetings of the Virtual Institute VH-VI-041 of the Helmholtz Association on Dense hadronic matter and $Q C D$ phase transition and the Helmholtz International Summer School Hot points in Astrophysics, Dubna, August 2-12, 2004. D.N.A. acknowledges support from Landesgraduiertenförderung Mecklenburg-Vorpommern, the work of V.Y. was supported in part by DFG under grant No. RUS $117 / 37 / 03$, by the Dynasty Foundation and by RFBR grant No. 05-02-16699. D.B. and M.B. thank for partial support of the Department of Energy during the program INT-04-1 on QCD and Dense Matter: From Lattices to Stars at the University of Washington, where this project has been initiated.
[1] R. Rapp, T. Schäfer, E. V. Shuryak and M. Velkovsky, Phys. Rev. Lett. 81, 53 (1998)

[2] M. G. Alford, K. Rajagopal and F. Wilczek, Phys. Lett. B 422, 247 (1998)

[3] D. Blaschke and C. D. Roberts, Nucl. Phys. A 642 (1998) 197.

[4] B.C. Barrois, Nucl. Phys. B129 (1977) 390.

[5] D. Bailin and A. Love, Phys. Rep. 107 (1984) 325.

[6] M. G. Alford, Ann. Rev. Nucl. Part. Sci. 51 (2001) 131.

[7] M. Buballa, Phys. Rep. 407 (2005) 205.

[8] A. Schmitt, Phys. Rev. D 71 (2005) 054016.

[9] S. B. Rüster, V. Werth, M. Buballa, I. A. Shovkovy and D. H. Rischke, arXiv:hep-ph/0503184

[10] D. Blaschke, S. Fredriksson, H. Grigorian, A. M. Öztas and F. Sandin, arXiv:hep-ph/0503194

[11] F. Neumann, M. Buballa and M. Oertel, Nucl. Phys. A 714, 481 (2003).

[12] M. Oertel and M. Buballa, arXiv:hep-ph/0202098

[13] M. Alford and K. Rajagopal, JHEP 0206 (2002) 031.

[14] C. Gocke, D. Blaschke, A. Khalatyan and H. Grigorian, arXiv:hep-ph/0104183

[15] H. Grigorian, D. Blaschke and D. N. Aguilera, Phys. Rev. C 69, 065802 (2004).

[16] I. Shovkovy, M. Hanauske and M. Huang, Phys. Rev. D
67, 103004 (2003).

[17] D. N. Aguilera, D. Blaschke and H. Grigorian, Nucl. Phys. A (2005) in press, arXiv:hep-ph/0412266

[18] D. Blaschke, T. Klähn and D. N. Voskresensky, Astrophys. J. 533 (2000) 406.

[19] D. Page, M. Prakash, J. M. Lattimer and A. Steiner, Phys. Rev. Lett. 85 (2000) 2048.

[20] D. Blaschke, H. Grigorian and D. N. Voskresensky, Astron. Astrophys. 368 (2001) 561.

[21] H. Grigorian, D. Blaschke and D. Voskresensky, Phys. Rev. C (2005) in press; arXiv:astro-ph/0411619

[22] T. Schäfer, Phys. Rev. D 62 (2000) 094007.

[23] M. G. Alford, J. A. Bowers, J. M. Cheyne and G. A. Cowan, Phys. Rev. D 67 (2003) 054018.

[24] A. Schmitt, I. A. Shovkovy and Q. Wang, arXiv:hep-ph/0502166

[25] M. Buballa, F. Neumann, M. Oertel and I. Shovkovy, Phys. Lett. B 595, 36 (2004).

[26] D. Blaschke, D. M. Sedrakian and K. M. Shahabasian, Astron. Astrophys. 350 (1999) L47.

[27] A. Schmitt, Q. Wang and D. H. Rischke, Phys. Rev. Lett. 91 (2003) 242301. 